\title{
PATHOPHYSIOLOGY OF DEPRESSION AND NOVEL SOURCES OF PHYTOCHEMICALS FOR ITS TREATMENT - A SYSTEMATIC REVIEW
}

\author{
D. Bakalov', R. Hadjiolova'1 , D. Pechlivanova² \\ ${ }^{1}$ Department of Pathophysiology, Medical University - Sofia, Bulgaria \\ ${ }^{2}$ Institute of Neurobiology, Bulgarian Academy of Sciences - Sofia, Bulgaria
}

\begin{abstract}
The rising burden of depression, which will soon be the second most common cause of disability in the world, is requesting new ways to treat and prevent it. Due to high number of significant adverse drug reactions of the conventional treatment, the modern pharmaceutical industry is more often turning their focus to novel plant-based solutions. We performed literature research based on standard literature search engines - PubMed, Google Scholar, Science Direct. A standard set of keywords related to our topic e.g. "Depression", "Mesembrine type alkaloids", "Narcissus" was used. The review describes the classical monoamine theory of depression and connects it with the newly found biochemical, genetic and morphological alterations associated with the major depressive disorder. The purpose of this review is to highlight the most important aspects of the pathophysiology of depression and to explore the possibilities to use mesembrine-like alkaloids isolated from Narcissus cv. Hawera in its treatment. We describe their effect on brain biochemistry and possible future investigations.
\end{abstract}

Key words: depression, mesembrine type alkaloids, Narcissus, phytomedicine

Corresponding author: Dimitar Bakalov, MD, Assistant Professor, Department of Pathophysiology, Medical University of Sofia, 2 Zdrave Str., 1431 Sofia, Bulgaria; e-mail: dbakalov@medfac.mu-sofia.bg

\section{INTRODUCTION}

A $\mathrm{s}$ depression is becoming one of the leading causes of disability, new ways for treatment are sought after. The researchers are turning their attention to different plant-derived substances. Mesembrine type alkaloids (Fig. 1) have a long history of traditional use, but recently the attention of scientists was concentrated on their possible use as a mood stabilizer and antidepressant in people with anxiety, stress and major depressive disorder. These type alkaloids are found in high concentrations in species of the genus Sceletium, some species of the genus Narcissus (Amaryllidaceae) and cultivated Narcissus cv. "Hawera" (Fig. 2). Literature data show that mesembrine alkaloids exerted an antidepressant-like effects on the brain. This leads to a hypothesis that the mesembrine fraction from Narcissus cv. "Hawera" (MZM) will exhibit the same anxiolytic and antidepressant effects.

\section{METHODOLOGY}

We performed literature research based on standard literature search engines - PubMed, Google Scholar, ScienceDirect. We used a set of keywords related to our topic e.g. "Depression", "Mesembrine type alkaloids", "Narcissus". We aimed to make a systematic review of the usage of mesembrine type phytochemicals in depression and the novel sources for their acquisitions. 

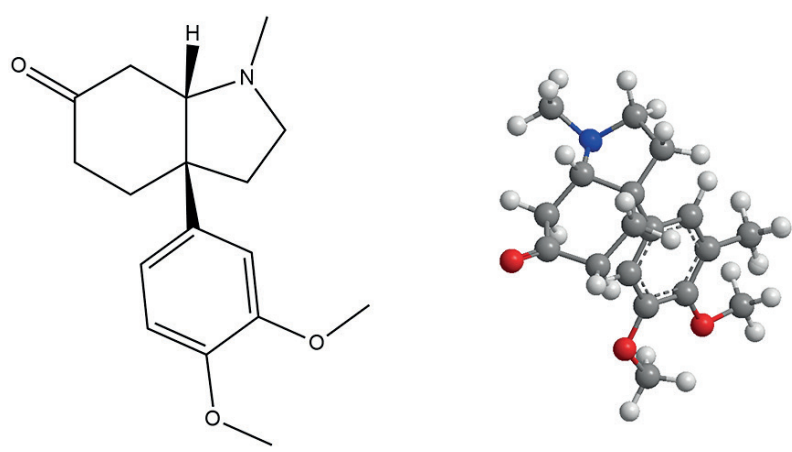

Fig. 1. Chemical structure of mesembrine

\section{CLINICAL DEPRESSION - EPIDEMIOLOGY AND DEFINITION}

Depression is affecting over 120 million people worldwide. The epidemiological data suggests that in the general populations there is a lifetime risk for developing depression between $10 \%$ to $15 \%$ [1]. Depression is the second most important factor causing disability and health issues in the world. It is also the leading cause of suicide, causing more than 128000 suicides just in the European region. According to the WHO in 2015, 44.3 million people were suffering from depression in Europe, with a frequency between $3.8 \%$ to $6.8 \%$ in different countries [2]. According to a survey conducted by the Bulgarian National Center of Public Health and Analysis (NCPHA), the most common psychiatric disease in Bulgaria is anxiety disorder (11.4\%) and in the second place - affective disorders (6.2\%) [3].

Clinical depression is known for its broad spectrum of symptoms and variable presentation. The three major dimensions of the disorder, are (1) anxiety-physical agitation-somatization; (2) depressed mood-motor retardation; and (3) hostility-interpersonal sensitivity $[4,5]$. The diagnostic conundrum of depression lies in the fact that in each affected individual those symptoms combine uniquely. The most commonly used diagnostic criteria are collected in the Diagnostic and Statistical Manual of Mental Disorders (DSM-5). The DSM- 5 outlines the following criterion to make a diagnosis of depression: "Depressed mood nearly every day, diminished interest or pleasure in almost all activities, significant weight loss when not dieting, slowing down of thought and a reduction of physical movement, fatigue, feelings of worthlessness or inappropriate guilt, diminished ability to think, recurrent thoughts of death, recurrent suicidal ideation". To receive a diagnosis of depression the individual must be experiencing five or more symptoms during the same 2-week period and at least one of the symptoms should be either (1) depressed mood or (2) loss of interest or pleasure. In patients with depression, the comorbidity of anxiety disorder (panic disorder, generalized anxiety disorder), post-traumatic stress disorder, OCD or personality disorder is common [6]. Physical illness is often accompanied by an increased risk of depressive illness. There are two general mech- anisms which can be the reason for that. The first one is the psychological or cognitive mechanism. A severe or chronic illness, with the threat it poses to one's life, may be the event that triggers a depressive episode in a predisposed individual. There are both genetic and nongenetic factors involved, as shown by twin studies [7, $8,9]$. As this mechanism is very nonspecific we should also consider that there is a significant biological basis behind depression, and biological alterations caused by physical illness can affect the depressive reaction. In addition, minor depression, mood changes, and depressive symptoms coexist with other manifestations of human distress. Such somatic presentations test the conventional distinction between physical and mental disorders and are a perpetual source of controversy [10].

\section{PATHOPHYSIOLOGY OF DEPRESSION}

One of the first mechanisms for the pathogenesis of depression proposed was the monoamine hypothesis, from nearly 50 years ago [11]. This hypothesis states that the patients with depression have decreased levels of serotonin (5-HT), noradrenaline (NA) and dopamine (DA) in the central nervous system (CNS) $[12,13,14,15]$. The first empirical proof of this hypothesis was the fact that some patients, treated with reserpine, developed clinical depression. The depression diminished after cessation of the treatment or electro-convulsive therapy [15]. This presentation was replicated in laboratory animals, treated with reserpine [13]. It was determined that reserpine significantly decreases the concentrations of the monoamines $-5 \mathrm{HT}$ and catecholamines $[16,17$, 18]. A lot of studies failed to find a confirmation for this theory, by pinpointing the specific system affected. The main problem was the inability to directly measure the brain monoamines. Even though the main antidepressant groups are becoming increasingly effective, the results are reversible. This is known as antidepressant therapy (ADT) tachyphylaxis and was at first recognized in patients treated with monoamine oxidase inhibitors (MAOls). Those patients responded poorly to the amendment of the therapy and got worse after the relapse compared to the pretreatment period [19]. In 1995, Fava et al. [20] found that one-third of the patients who had reached full remission on SSRI experienced a relapse (ADT tachyphylaxis) between 3 months and 1 year later despite following the therapeutic plan. Some studies even show that major depressive disorder (MDD) patients who developed ADT tachyphylaxis can become less responsive to therapy [21]. At the same time, monoamine depletion does not increase the severity of the symptoms of patients without treatment, nor does it cause clinically significant depression in healthy volunteers with no depressive illness. Those finding put a question mark on the precise role that a deficiency in the monoamine system may play in depression itself [14]. Today it is known that the pathobiochemistry behind depression is a significantly more complex 
problem affecting both the neurotransmitter systems and the morphology of the brain.

In 1997, Drevets et al. [22] showed a link between the affective disorders and reduced cortical volume. This gave a reason for the investigation of the connection between the pathogenesis of depression and morphological alterations in the CNS [23]. In many of the tested subjects, there was a repeated finding - decreased thickness of the prefrontal cortex (PFC) and decreased total number of neurons [24]. Further studies have revealed a loss of synapses and dendrite density in the PFC [25] and hippocampus [26] of patients with depression. Pathologic changes in the hippocampus (Fig. 2), a part of the limbic system located in the medial temporal lobe, are often associated with various symptoms of depression such as cognitive decline and memory impairment [27].

One of the reasons for morphological changes in the hippocampus is prolonged stress [28]. In PTSD patients the hippocampal volume is significantly smaller, which correlated with deficits in short term memory [29]. A fairly recent imaging study in rats demonstrated that chronic restraint stress (CRS) does cause a reduction in hippocampal volume compared to the starting values [30]. In models of CRS, there was a significant reduction of the dendritic spines and microstructural alterations in the CA3 region [31] and suppresses the adult neurogenesis in the dentate gyrus [32].

Some of those ultrastructural changes can be explained by dysregulation of the nerve neurotrophic factors such as nerve growth factor, e.g. brain-derived neurotrophic factor (BDNF). Studies are showing that chronic mild stress causes downregulation of the production of BDNF in the dentate gyrus [33], which can be associated with the neuronal atrophy and decreased cell division. Antidepressant therapy, like serotonin and norepinephrine reuptake inhibitors (SSRI or SNRI) upregulates the levels of BDNF in the hippocampus [34, 35]. Also, BDNF has demonstrated

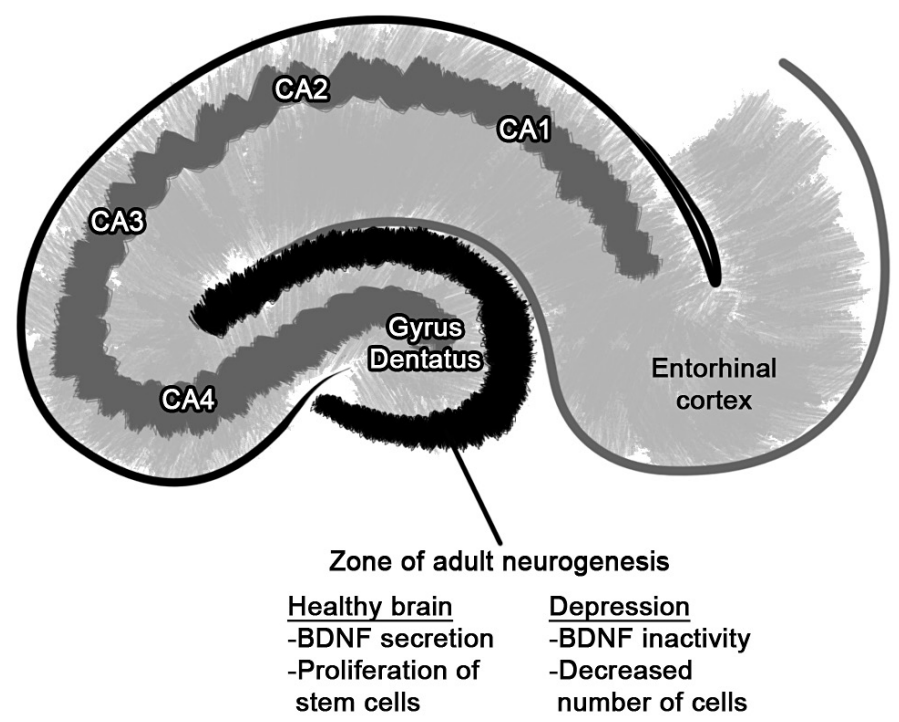

Fig. 2. Hippocampus anatomy and differences in depression antidepressant-like effects in behavioral rodent models of depression. This was shown after the infusion of BDNF into certain brain regions such as the hippocampus and PFC [36]. BDNF polymorphisms have also been suggested to play a role in familial depression, but replication results were inconclusive [37].

Another important group of molecules with a possible role in the pathogenesis of depression are the phosphodiesterases (PDE), especially PDE4, a highly abundant in the brain isotype, which functions as an important regulator of CAMP [38]. PDE4-targeted therapy was first focused on the treatment of COPD and respiratory disorders, but in vitro studies have shown promise in neuroinflammation-related diseases. The first CNS permeable PDE4 inhibitor is Rolipram, created with the intent to target neuroinflammation. Rolipram has shown promising results in in vivo models of depression, Alzheimer's disease, Parkinson's disease, and neuropathic pain [39, 40, 41]. PDE4 inhibitors suppress neuroinflammation by increasing the level of CAMP, promoting CREB phosphorylation, and inhibiting NF-KB activation in the cortex and hippocampus in mice subjected to LPS-induced neuroinflammation [42]. Furthermore, a study in mice challenged by LPS found that PDE4 inhibition caused anti-depressant effects as confirmed by the decrease in depressant-like behaviors with the duration of immobility in the forced swim and tail suspension tests [43].

\section{TREATMENT OF DEPRESSION - HISTORY AND NEW HORIZONS}

In hospital settings, $50 \%$ of all psychiatric consultations and $12 \%$ of all hospitalizations are related to clinical depression [44]. This is one of the reasons why there is active research for new and effective treatments. The contemporary pharmacotherapy for affective disorders is mainly focused on the regulation of the monoamine neuromediation. The main targets are 5-HT, NA, and DA.

The first drug of the antidepressant family was created by accident in the laboratories of Hoffmann-La Roche Ltd., USA. In this lab, a few years earlier was patented the very effective tuberculosis antibiotic - isonicotinylhydrazide (INH) [45]. In an attempt to increase the potency of the drug, Fox and Gibas (1953) created a new monoalkyl derivative - iproniazide, which in turn was the catalyst for developing the modern antidepressants [46]. During different clinical trials of the drug, the doctors observed unusual and unexpected adverse drug reactions (ADRs) - euphoria, increased appetite, hypomania. In 1957 Loomer et al. conducted a systematic clinical trial to test the effects of iproniazid on patients with depression. In $70 \%$ of the cases, there was a significant improvement in the symptoms [47]. Iproniazide was the first monoamine oxidase inhibitor in the history. Based on this monoamine theory 
during the following years, many more drug classes were developed and refined. Another group of molecules with antidepressant effect were the tricyclic antidepressants (TCAs). It was suggested that TCAs, like MAOls, excert their beneficial psychotropic effect by increasing synaptic serotonin and catecholamine concentrations [48].

As we mentioned the inflammatory processes can have significant role in the aetiology of depression, as shown by animal models, and this was again supported with new literature data demonstrating that the SSRIs possess significant anti-inflammatory activities by reducing pro-inflammatory cytokines, and also a role in promoting neurogenesis [49]. Based on this monoamine theory during the following years, many more drug classes were developed and refined. SSRIs remain the primary pharmacological option for clinicians in the treatment of depression.

Despite the higher specificity and better safety profile, the conventional psychopharmacotherapy is still associated with a high number of ADRs, which is especially significant in the patients with depression, because they often have co-morbidities. The frequencies of chronic disorders in depression are as follows: asthma $-27 \%$ [50], atopic dermatitis $-5 \%$ [51], COPD - 24.6\% [52], gout associated arthritis $20 \%$ [53], rheumatoid arthritis - 15\% [54], systemic lupus - 22\% [53], and stroke - 30\% [55]. The already mentioned high rate of ADT tachyphylaxis $(33 \%)$ is another problem that should be addressed, in the search for new, more effective and complex treatment options.

It is not always possible to find the balance between the positive therapeutic effect and its ADRs, which is the reason why many patients are seaking for alternative treatment options such as phytomedicine as an addition to the conventional drugs. Some of those

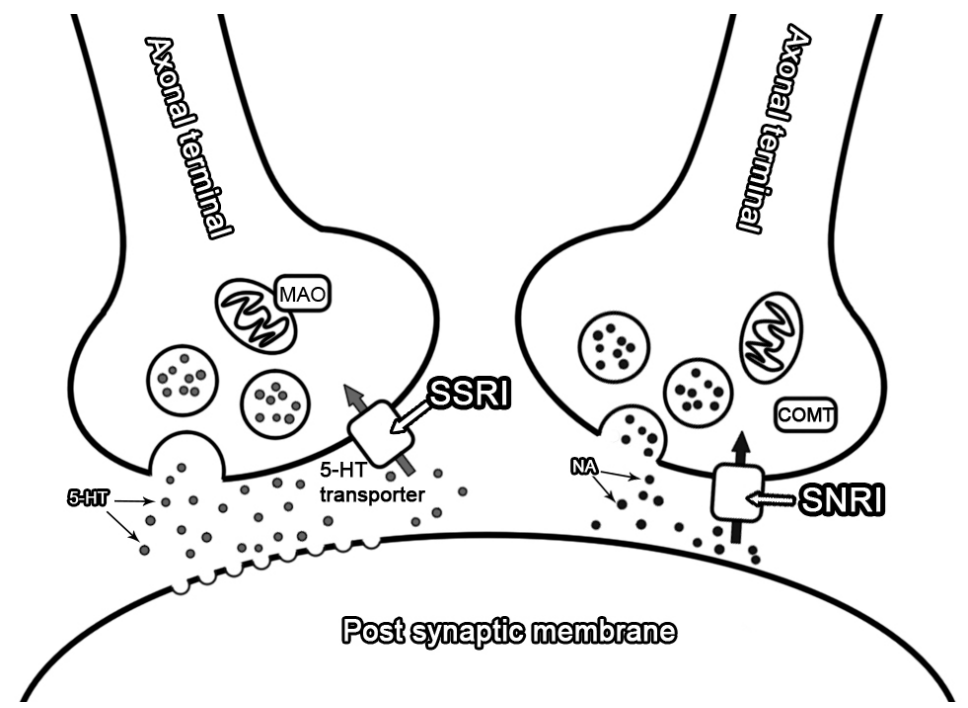

Fig. 3. Presynaptic cleft. 5-HT - 5-hydroxytryptamine; COMT - Catechol-O-methyltransferase; NA - noradrenaline; MAO - monoamine oxidase; SNRI - serotoninnorepinephrine reuptake inhibitors e.g. Duloxetine; SSRI - selective serotonin reuptake inhibitor e.g. Paroxetine "alternative" plant-based treatments were described in the ancient times, ever since Hippocrates (460379 BC) described depression for the first time, as "melancholia", derived from the Ancient Greek melas, "black", and kholé, "bile" [56].

Today there is a significant increase in the usage of phytomedicine. A survey conducted in the lates $90 \mathrm{~s}$ showed that $57 \%$ of the anxiety patients and $54 \%$ of those with depression have used plant-based drugs or alternative medicine in the last 12 months [57].

The modern phytotherapy is still a very young branch of pharmacology, but there is an exponential increase in the number of studies and breakthroughs in recent years. Besides the classic powerful alkaloids isolated from plants like Erythroxylon coca - cocaine, Papaver somniferum - morphine, Areca catechu - arecoline, in the last decades many more plants show potential for becoming a source of therapeutic molecules e.g. St John's wort (Hypericum perforatum), Sceletium tortuosum and the genus Narcissus [57, 58].

An important aspect of phytotherapy is the fact that often it is impossible to isolate a single active molecule defining the pharmacodynamics of the extract. The action is frequently attributed to the synergistic effects of different molecules found in the plant [60].

Some plants are known for their effectiveness in modifying the course of the depression by acting on the monoamine neurotransmission, such as $\mathrm{H}$. perforatum, Rhodiola rosea, Crocus sativus [61, 62]. Others act on GABA, cytokines and even - opioid and cannabinoid systems [62]. A meta-analysis from 2009 showed that $H$. perforatum is more effective than placebo, and also showed a better safety profile compared to a conventional SSRIs - significantly less treatment seizing due to adverse drug reactions [63].

\section{NARCISSUS PLANTS IN MEDICINE}

Narcissus is a member of the Amaryllidaceae family, which comprises 65 genera and more than 860 species. These species are broadly dispersed in tropical, subtropical, and temperate areas of the northern and southern hemispheres [59, 64, 65]. They are praised for their decorative value and have been used in medicine since ancient times [66, 67]. The genus Narcissus has been targeted by researchers as a new source of biomedically active alkaloids, and isolate more than 200 alkaloids from the plants of this family $[59,65]$. Within this group, the genus Narcissus is used in traditional medicine to treat a variety of health problems. A range of pharmacological effects has been attributed to these alkaloids, and include antiviral, antifungal, and antitumor activity $[68,65]$. There is a large number of phytochemical studies on Narcissus 
evaluating their possible use as a source for neuroactive substances, for example as a source of galantamine [59], an alkaloid with acetylcholine esterase inhibitory activity, used as a treatment for cognitive decline in mild Alzheimer's disease [69]. In our studies, the focus was pointed at the mesembrine alkaloids isolated from Narcissus cv. Hawera, and their potential antidepressant properties.

\section{MESEMBRINE ALKALOIDS}

This interesting group of compounds was first discovered around the same time iproniazide was synthesized, in 1957 [70]. Plants containing mesembrine alkaloids have been used for centuries. The most popular plant is Sceletium tortuosum, which has a long history of use in traditional South African medicine as a moodaltering substance from prehistoric times [58]. It was first described on paper in 1662 by Jan van Riebeeck. The Sceletium plant was chew, made in teas and tinctures, used as a snuff and smoked [71]. Through the use of in vitro studies, mesembrine-like alkaloids demonstrated SSRI activity, which suggested the possible use in depression, mood disorders and OCD [72]. Synthetic mesembrine alkaloids have prominent inhibitory activity on the serotonin transporter, with possible modulatory effects on norepinephrine and dopamine [73]. Additionally, mesembrine has also been shown to inhibit phosphodiesterase-4 (PDE4) [74]. As mentioned earlier, PDE4 inhibitors are currently a new and promising area for research for a new treatment for depression. By altering the levels of cyclic AMP (cAMP), the PDE4 family of enzymes has a direct role in the pathogenesis of MDD and it has been shown that selective inhibition of these enzymes can demonstrate noticeable antidepressant activity [75]. It was also shown that PDE4 inhibitors enhance the induction of BDNF mRNA in rat hippocampus [76], thus, as we discussed previously, by promoting this neurotrophic factor we can have a positive antidepressant effect in affected individuals.

Until now there were no ADRs reports concerning mesembrine alkaloids preparations. In a study conducted to test their safety, extract from Sceletium was given to seven healthy beagles and one dog with dementia at a dose of $10 \mathrm{mg} / \mathrm{kg}$ twice per day for six days. After extensive clinical and paraclinical investigation, including $\mathrm{CBC}$, biochemistry, and kidney function tests it was reported that there were no significant changes and no behavioral abnormalities.

A study conducted to analyze the effects of Zembrin, a drug that contains a standardized extract of Sceletium tortuosum, showed that after treatment with one dose of $25 \mathrm{mg}$ the amygdala's reaction to fearful facial expressions was ameliorated. This was visualized with functional-MRI, which also showed an attenuation of the amygdala-hypothalamus coupling. This proves that the SSRI capabilities combined with the PDE4 action of Sceletium may be used not only for the treatment of depression but also in anxiety disorders, which often accompanies MDD.

\section{CONCLUSION}

Major depressive disorder is one of the leading cause of disability in the world. The cause is believed to be a combination of genetic, environmental, and psychological factors. This is the reason why rational treatment is still a significant medical conundrum, and the effectiveness varies between patients. This review covers the general pathophysiological mechanisms of depression and summarizes the usage of mesembrine-like alkaloids - plant-derived substances, as antidepressants. Additionally, we report the possible use of the mesembrine alkaloids isolated from Narcissus cv. Hawera, and their potential antidepressant properties.

Acknowledgements. This work was supported by the Medical Science Council, Medical University, Sofia, Bulgaria, contract № 69/2019

\section{REFERENCES}

1. Demyttenaere K, Bruffaerts R, Posada-Villa J, et al. Prevalence, severity, and unmet need for treatment of mental disorders in the World Health Organization World Mental Health Surveys. JAMA. 2004; 291:2581-2590.

2. WHO, Fact Sheet - Mental Health 2019, Accessed 04.03.2019 at: http://www.euro.who.int/en/health-topics/noncommunicablediseases/mental-health/data-and-resources/fact-sheet-mentalhealth-2019.

3. Hinkov H, Dimitrov $P$, Zarkov Z et al. National Representative Epidemiological Study Of Common Mental Disorders In Bulgaria Epibul 2, 2016-2017: Tool, Methodology, Process Evaluation. Soc Med. 2017; 1: 21-23.

4. Katz MM, Koslow SH, Berman $\mathrm{N}$ et al. A multi-vantaged approach to measurement of behavioral and affect states for clinical and psychobiological research. Psychol Rep. 1984; 55:619-671.

5. Katz MM, Maas JW. Psychopharmacology and the etiology of psychopathological states: are we looking in the right way? Neuropsychopharmacology. 1994; 10:139-144.

6. Mimura M. Comorbidity of Depression and Other Diseases. JMAJ 2001; 44(5): 225-229.

7. Kendler KS, Neale M, Kessler R et al. A twin study of recent life events and difficulties. Arch Gen Psychiatry. 1993; 50:789-796.

8. Kendler KS, Gardner CO, Prescott CA. Toward a comprehensive developmental model for major depression in women. Am J Psychiatry. 2002; 159:1133-1145.

9. Kendler KS, Thornton LM, Gardner CO. Genetic risk, number of previous depressive episodes, and stressful life events in predicting onset of major depression. Am J Psychiatry. 2001; 158:582-156.

10. Goodwin GM. Depression and associated physical diseases and symptoms. Dialogues Clin Neurosci. 2006; 8(2):259-265.

11. Schildkraut JJ. The catecholamine hypothesis of affective disorders: a review of supporting evidence. Am J Psychiatry. 1965; 122(5):509-22.

12. Bunney WE Jr, Davis JM. Norepinephrine in depressive reactions. A review. Arch Gen Psychiatry. 1965; 13(6):483-94.

13. Hirschfeld RM. History and evolution of the monoamine hypothesis of depression. J Clin Psychiatry. 2000; 61 S6:4-6.

14. Delgado PL. Depression: the case for a monoamine deficiency. J Clin Psychiatry. 2000; 61 S6:7-11.

15. Muller JC, Pryor WW, Gibbons JE, Orgain ES. Depression and anxiety occurring during Rauwolfia therapy. J Am Med Assoc. 1955; 29; 159(9):836-9.

16. Shore PA, Silver SL, Brodie BB.Interaction of reserpine, serotonin, and lysergic acid diethylamide in brain. Science. 1955; 122(3163):284-5.

17. Shore PA, Pletscher A, Tomich EG et al. Role of brain serotonin in reserpine action. Ann N Y Acad Sci. 1957; 66(3):609-15; discussion, 615-7.

18. Weiner N, Cloutier G, Bjur R, Pfeffer RI. Modification of norepinephrine synthesis in intact tissue by drugs and during short-term adrenergic nerve stimulation. Pharmacol Rev. 1972; 24(2): 203-221.

19. Mann JJ. Loss of antidepressant effect with long-term monoamine oxidase inhibitor treatment without loss of monoamine oxidase inhibition. J Clin Psychopharmacol. 1983; 3:363-366. 
20. Fava M, Rappe SM, Pava JA, et al. Relapse in patients on longterm fluoxetine treatment respond to increased fluoxetine dose. J. Clin Psychiatry. 1995; 56:52-55.

21. Sharma V. Loss of response to antidepressants and subsequent refractoriness: diagnostic issues in a retrospective case series. $J$ Affect Disord. 2001; 64:99-106.

22. Drevets WC, Price JL, Simpson JR Jr et al. Subgenual prefrontal cortex abnormalities in mood disorders. Nature. 1997; 386:824-827.

23. Russo SJ, Nestler EJ. The brain reward circuitry in mood disorders. Nat Rev Neurosci. 2013; 14:609-625.

24. Rajkowska G, Miguel-Hidalgo JJ, Wei J et al. Morphometric evidence for neuronal and glial prefrontal cell pathology in major depression. Biol Psychiatry. 1999; 45:1085-1098.

25. Kang HJ, Kim JM, Lee JY et al. BDNF promoter methylation and suicidal behavior in depressive patients. J Affect Disord. 2013; 151:679-685.

26. Duric V, Banasr M, Stockmeier CA et al. Altered expression of synapse and glutamate related genes in postmortem hippocampus of depressed subjects. Int J Neuropsychopharmacol. 2013; 16:69-82.

27. 2Morales-Medina JC, Juarez I, Venancio-Garcia E et al. Impaired structural hippocampal plasticity is associated with emotional and memory deficits in the olfactory bulbectomized rat. Neuroscience. 2013; 236:233-243

28. Willner $P$, Scheel-Krüger J. The neurobiology of depression and antidepressant action. Neuroscience and Biobehavioral Reviews 2013; 37: 2331-2371

29. Bremner JD, Randall P, Scott TM et al. MRI-based measurement of hippocampal volume in patients with combat-related posttraumatic stress disorder. Am J Psychiatry. 1995; 152: 973-981.

30. Lee T, Jarome T, Li SJ, Kim JJ, Helmstetter FJ. Chronic stress selectively reduces hippocampal volume in rats: a longitudinal magnetic resonance imaging study. Neuroreport. 2009; 20: 1554-1558.

31. Conrad CD, LeDoux JE, Magarinos AM, McEwen BS. Repeated restraint stress facilitates fear conditioning independently of causing hippocampal CA3 dendritic atrophy. Behav Neurosci. 1999; 113: $902-913$

32. Schoenfeld TJ, Gould E. Stress, stress hormones, and adult neurogenesis. Exp Neurol. 2012; 233:12-21.

33. Grønli J, Bramham C, Murison R et al. Chronic mild stress inhibits BDNF protein expression and CREB activation in thedentate gyrus but not in the hippocampus proper. Pharm Bio Behav. 2006; 85(4):842-9.

34. Duman RS, Heninger GR, Nestler EJ. A molecular and cellular theory of depression. Arch. Gen. Psychiatry. 1997; 54(7):597-606.

35. Duman RS, Monteggia LM. A neurotrophic model for stress-related mood disorders. Biol. Psychiatry. 2006; 59(12):1116-1127.

36. Björkholm C, Monteggia LM. BDNF - a key transducer of antidepressant effects. Neuropharmacology. 2016; 102:72-79. doi:10.1016/j.neuropharm.2015.10.034

37. Levinson, D. The genetics of depression: a review. Biological Psychiatry. 2006; 60 (2): 84-92.

38. Maurice, $\mathrm{DH}, \mathrm{Ke}, \mathrm{H}$, Ahmad, $\mathrm{F}$ et al. Advances in targeting cyclic nucleotide phosphodiesterases. Nat. Rev. Drug Discov. 2014; 13 290-314.

39. Rose, GM, Hopper, A, De Vivo, M, Tehim, A. Phosphodiesterase inhibitors for cognitive enhancement. Curr. Pharm. Des. 2005; 11:3329-3334

40. García-Osta, A, Cuadrado-Tejedor, M, Garcia-Barroso, C et al. Phosphodiesterases as therapeutic targets for Alzheimer's disease. ACS Chem. Neurosci. 2012; 3:832-844.

41. Pearse, DD, Hughes, ZA. PDE4B as a microglia target to reduce neuroinflammation. Glia. 2016; 64:1698-1709.

42. Zou, ZQ, Chen, JJ, Feng, HF et al. Novel phosphodiesterase 4 inhibitor FCPR03 alleviates lipopolysaccharide-induced neuroinflammation by regulation of the CAMP/PKA/CREB signaling pathway and NF-kappaB Inhibition. J. Pharmacol. Exp. Ther. 2017; 362, 67-77.

43. Yu, H, Zou, Z, Zhang, X et al. Inhibition of phosphodiesterase 4 by FCPR03 alleviates lipopolysaccharide-induced depressive-like behaviors in mice: involvement of p38 and JNK signaling pathways. Int. J. Mol. Sci. 2018; 19: E513.

44. Kuo DC, Tran M, Shah AA, et al. Depression and the suicidal patient. Emerg Med Clin North Am 2015; 33:765-78.

45. Riede, HL. Fourth-generation fluoroquinolones in tuberculosis. Lancet. 2009; 373 (9670): 1148-1149

46. Fox H, Gibas T. Synthetic tuberculostats. V. Alkylidene derivatives of isonicotinyhydrazineJ. Org. Chem. 1953; 18(8): 983-989

47. Loomer, HP, Saunders, JC, Kline, NS. A clinical and pharmacodynamic evaluation of iproniazid as a psychic energizer. Psychiatric Research Reports. 1957; 8:129-141.

48. Chockalingam R, Gott BM, Conway CR. Tricyclic Antidepressants and Monoamine Oxidase Inhibitors: Are They Too Old for a New Look? Handbook of Experimental Pharmacology. 2019;250:37-48.

49. Walker FG. A critical review of the mechanism of action for the selective serotonin reuptake inhibitors: Do these drugs possess anti-inflammatory properties and how relevant is this in the treatment of depression? Neuropharmacology, 2013; 67: 304-317.

50. Lu Y, Mak KK, Van Bever HP, et al Prevalence of anxiety and depressive symptoms in adolescents with asthma: a meta-analysis and meta-regression. Pediatr Allergy Immunol. 2012; 23:707-715.

51. Lim VZ, Ho RC, Tee SI, et al. Anxiety and depression in patients with atopic dermatitis in a Southeast Asian tertiary dermatological center. Ann Acad Med Singapore 2016.

52. Zhang MW, Ho RC, Cheung MW, et al. Prevalence of depressive symptoms in patients with chronic obstructive pulmonary disease: a systematic review, meta-analysis and meta-regression. Gen Hosp Psychiatry 2011; 33:217-23.

53. Mak A, Tang CS, Chan MF, et al. Damage accrual, cumulative glucocorticoid dose and depression predict anxiety in patients with systemic lupus erythematosus. Clin Rheumatol 2011.

54. Ho RC, Fu EH, Chua AN, et al. Clinical and psychosocial factors associated with depression and anxiety in Singaporean patients with rheumatoid arthritis. Int J Rheum Dis 2011; 14:37-47.

55. Mak KK, Kong WY, Mak A, et al. Polymorphisms of the serotonin transporter gene and post-stroke depression: a meta-analysis. J Neurol Neurosurg Psychiatry 2013.

56. Liddell, Henry and Robert Scott (1980). A Greek-English Lexicon (Abridged Edition). United Kingdom: Oxford University Press. ISBN 0-19-910207-4.

57. Kessler, RC, Soukup, J, Davis, RB et al. The use of complementary and alternative therapies to treat anxiety and depression in the United States. Am. J. Psychiatry. 2001; 158:289-294.

58. Harvey, AL, Young, LC, Viljoen, AM, Gericke, NP. Pharmacological actions of the South African medicinal and functional food plant Sceletium tortuosum and its principal alkaloids. Journal of Ethnopharmacology. 2011; 137(3):1124-1129.

59. Hanks, GR. (ed.). Narcissus and daffodil: The genus Narcissus. CRC Press, Boca Raton, FL, 2003.

60. Heinrich, M, Barnes, J, Gibbons, S, Williamson, E. Fundamentals of Pharmacognosy and Phytotherapy. Churchill Livingstone, London, 2004

61. Kumar, V. Potential medicinal plants for CNS disorders: an overview. Phytother. Res. 2006; 20(12):1023-1035.

62. Spinella, M. The Psychopharmacology of Herbal Medicine: Plant Drugs That Alter Mind, Brain and Behavior (Paperback). MIT Press, Cambridge, 2001.

63. Rahimi, R, Nikfar, S, Abdollahi, M. Efficacy and tolerability of Hypericum perforatumin major depressive disorder in comparison with selective serotonin reuptake inhibitors: a meta-analysis. Prog Neuropsychopharmacol Biol Psychiatry. 2009; 33:118-127.

64. Buket B, Ahmet E, Gulen K et al. Somer. Alkaloid Profiling of Galanthus woronowii Losinsk. by GC-MS and evaluation of its biological activity. Marmara Pharmaceutical Journal. 2017; 21 (4):915-920.

65. Berkov, S, Osorio E, Viladomat F, Bastida J. Chapter Two - Chemodiversity, chemotaxonomy and chemoecology of Amaryllidaceae alkaloids. The Alkaloids: Chemistry and Biology. 2020; 83: 113-185

66. Artyushenko, ZT. Amaryllidaceae of USSR, morphology, taxonomy and use. Nauka, Leningrad, Russia, 1970.

67. Khamidkhodzhaev, SA. Biology, resources and introduction of medicinal plants of Ungernia Bunge and Ficus L. in Middle Asia. Main Botanical Garden, Moscow, Russia, Dr. Sci. Diss. Abstr, 1984.

68. Pigni, NB, Berkov, S, Elamrani, A, et al. Two New Alkaloids from Narcissus serotinus L.. Molecules, 2010; 15: 7083-7089.

69. Birks, J Birks, Jacqueline S. Cholinesterase inhibitors for Alzheimer's disease. The Cochrane Database of Systematic Reviews. 2006; 1: CD005593.

70. Bodendorf, K, Krieger, W. Alkaloids of Mesembryanthemum tortuosum. Arch. Pharm. 1957; 290:441.

71. Smith, MT, Crouch, NR, Gericke, N, Hirst, M. Psychoactive Constituents of the Genus Sceletium N.E.Br. and other Mesembryanthemaceae: A Review. Journal of Ethnopharmacology. 1996; 50(3):119-130.

72. Gericke, N, Van Wyk, B. In African Natural Health CC(Ed.), Pharmaceutical compositions containing mesembrine, 2001.

73. Harvey, A. In Gericke N. (Ed.), Personal communication to Nigel Gericke, 2008

74. Napoletano M, Norcini G, Pellacini F et al. Phthalazine PDE4 inhibitors. Part 2: the synthesis and biological evaluation of 6-methoxy-1,4-disubstituted derivatives. Bioorg Med Chem Lett. 2001;11(1):33-7.

75. Houslay, M, Schafer, P, Zhang, K. Keynote review: Phosphodiesterase-4 as a therapeutic target. Drug Discovery Today. 2005; 15:11503.

76. Fujimaki, K, Morinobu, S, Duman, $R$. Administration of a cAMP Phosphodiesterase 4 Inhibitor Enhances Antidepressant-Induction of BDNF mRNA in Rat Hippocampus. Neuropsychopharmacol, 2000; 22: 42-51.

Received: 25 April 2020, Accepted :07 June 2020 